Book Chapter

\title{
Eco-Friendly Coatings Containing Microcapsules with Fire-Resistant Properties
}

Andrés Felipe Jaramillo ${ }^{1}$, Andrés Díaz-Gómez ${ }^{2}$, Jesús Ramirez $^{2}$, María Elizabeth Berrio ${ }^{2}$, Vanessa Cornejo ${ }^{2}$, David Rojas $^{2}$, Luis Felipe Montoya ${ }^{2}$, Adriana Mera $^{3}$ and Manuel Francisco Melendrez ${ }^{2,4 *}$

${ }^{1}$ Departament of Mechanical Engineering, Universidad de La Frontera, Chile

${ }^{2}$ Interdisciplinary Group of Applied Nanotechnology (GINA), Hybrid Materials Laboratory (HML), Department of Materials Engineering (DIMAT), Faculty of Engineering, University of Concepcion, Chile

${ }^{3}$ Instituto de Investigación Multidisplinario en Ciencia y Tecnología, Universidad de La Serena, Chile

${ }^{4}$ Unidad de Desarrollo Tecnológico, Chile

*Corresponding Author: Manuel Francisco Melendrez, Interdisciplinary Group of Applied Nanotechnology (GINA), Hybrid Materials Laboratory (HML), Department of Materials Engineering (DIMAT), Faculty of Engineering, University of Concepcion, 270 Edmundo Larenas, Box 160-C, Concepcion 4070409, Chile

Published July 08, 2021

This book chapter is a fuller and more detailed publication of the article published by Manuel Francisco Melendrez, et al. at Coatings in February 2021. (Jaramillo, A.F.; Díaz-Gómez, A.; Ramirez, J.; Berrio, M.E.; Cornejo, V.; Rojas, D.; Montoya, L.F.; Mera, A.; Melendrez, M.F. Eco-Friendly Fire-Resistant Coatings Containing Dihydrogen Ammonium Phosphate Microcapsules and Tannins. Coatings 2021, 11, 280. https://doi.org/10.3390/coatings11030280) 
How to cite this book chapter: Andrés Felipe Jaramillo, Andrés Díaz-Gómez, Jesús Ramirez, María Elizabeth Berrio, Vanessa Cornejo, David Rojas, Luis Felipe Montoya, Adriana Mera, Manuel Francisco Melendrez. Eco-Friendly Coatings Containing Microcapsules with Fire-Resistant Properties. In: Alessio Bosio, editor. Surfaces, Interfaces and Coatings Technology. Hyderabad, India: Vide Leaf. 2021.

(C) The Author(s) 2021. This article is distributed under the terms of the Creative Commons Attribution 4.0 International License(http://creativecommons.org/licenses/by/4.0/), which permits unrestricted use, distribution, and reproduction in any medium, provided the original work is properly cited.

Author Contributions: A.D.G and M.E.B: Carried out the tannin extraction and its complete characterization. J.R and V.C: Designed the fire-resistance and intumescence experiments. L.F.M, A.F.J and M.E.B: Carried out the fireproof and intumescent formulations and the dual scheme. A.C.M, M.F.M: Analyzed the results and wrote the manuscript. A.D.G and J.R: Developed the field tests and consolidated the formulations obtained. All authors reviewed the manuscript and contributed to its consolidation. All authors have read and agreed to the published version of the manuscript.

Funding: This work was funded by ANID FONDEQUIP Project $\mathrm{N}^{\circ}$ EQM150139, PIA/APOYO CCTE AFB170007, FONDECYT Initiation 11190358 and project FONDEF IDEA ID17i10333.

Acknowledgments: The authors would like to thank to the Interdisciplinary Group of Advanced Nanocomposites (Grupo Interdisciplinario de Nanocompuestos Avanzados, GINA) of the Department of Engineering Materials (DIMAT, according to its Spanish acronym), Engineering School of the University of Concepción, for its laboratory of nanospectroscopy (LABNANOSPECT). AFJ would like to thank the University of La Frontera. National Agency for Research and Development of Chile (ANID) by project: FONDEQUIP Project ${ }^{\circ}$ EQM150139, PIA/APOYO CCTE AFB170007, FONDECYT Initiation 
11190358 and project FONDEF IDEA ID17i10333. MFM would like to thank Valentina Lamilla for her enormous support.

Conflicts of Interest: The authors declare no conflict of interest.

\section{Graphical Abstract}



\section{Abstract}

The effect of microencapsulation of dihydrogen ammonium phosphate (MAP) in the generation of fire-resistant coatings was studied in the presence of tannins extracted from Pinus radiata. MAP was encapsulated to avoid interaction with sodium carbonate $\left(\mathrm{Na}_{2} \mathrm{CO}_{3}\right)$, which upon contact with fire, generates unwanted gases. Thus, a fireproof (or intumescent) protective film was produced in the presence of the tannins. Microcapsules were polymerized with melamine and characterized by FTIR, TGA, SEM-EDS. The microcapsules were spherical with a diameter between 0.7 and $1 \mu \mathrm{m}$. The as-produced microcapsules were mixed with tannin extract and the properties of their film were evaluated on wood and structural steel substrates; their fire resistance on medium density fiberboard was also evaluated. Flame resistance tests showed a carbonization index of $26.86 \%$ using microcapsules $(3 \% \mathrm{w} / \mathrm{w})$; this is better than commercial coatings. The film properties were similar to commercial coatings, but the adherence was slightly decreased, due to agglomeration and also films flexibility. 
Surfaces, Interfaces and Coatings Technology

\section{Keywords}

Fire Resistant Coating; Tannin; Eco-Friendly; Microencapsulation; Dihydrogen Ammonium Phosphate

\section{Introduction}

Structural steel has been widely used in the construction industry due to properties such as high strength/weight ratio, high ductility, among others. However, its mechanical resistance decreases if the temperature exceeds $500{ }^{\circ} \mathrm{C}$ during a fire. Therefore, enhancing this property is a concern for the construction industry; also, technological solutions focused mainly on the development of foams and coatings have been proposed [1]. Fire-resistant coatings (fire retardant and intumescent) have been widely used to protect metal structures and even wood substrates. These types of fire-resistant coatings are composed of a carbon forming material, a mineral acid catalyst, a blowing agent, and a binder resin. During the combustion process, these components operate synergistically to form a honeycomb carbon structure that thermally isolates the coated steel substrate and establishes a protective barrier blocking heat transfer to the steel; while in wood, acts chemically to release less pyrolysis gasses and contain more of the fuel as thermally insulating char [2,3]. The mechanism of protection in fireproof coatings includes retarding the advance of flames on the substrate. On the other hand, in intumescent coatings, the acidic source can release $\mathrm{NH}_{3}$ and organic acids during the combustion process; this initiates an esterification reaction with the carbonaceous agent. The carbonaceous agent is carbonized by dehydration, and finally, the blowing agent forms an intumescent foam or layer on the surface of the polymeric material $[1,4]$. These coatings have numerous disadvantages: the carbon protective layer is vulnerable to high temperatures, the coating is prone to aging, and in water-based coatings, the coating displays poor adhesion to substrates $[5,6]$.

To achieve suitable fire resistance, coatings are generally formulated with a high content of solid fillers; these impair the mechanical properties and durability of the film. Moreover, a 
major disadvantage of additives is their poor compatibility with the coating resin; this significantly reduces the protection capacity in a fire. There has been limited use of microencapsulation technology in fire retardants and intumescent coatings. Almost no research has been conducted on the durability and fire resistance of the flame-retardant coating after the static immersion test. To overcome these problems, the use of microencapsulated dihydrogen ammonium or monoammonium phosphate (MAP) (acid source) as a fireresistant additive has been an effective strategy $[7,8]$.

For the scientific and industry communities, the development of ecological and sustainable fire-resistant coating systems has become an urgent challenge. Recently, the study of products from natural sources as potential additives has garnered considerable attention. Their characteristics allow the generation of an insulating carbon layer on substrate surfaces. They provide efficient fire resistance functionality and are therefore promising alternatives to halogen-based chemicals. Almost all renewable resource-based additive approaches to form fire-resistant coatings can impart these beneficial properties $[9,10]$. Modifying the additive properties through microencapsulation with water or insoluble polymers is an effective method to improve water resistance and polarity. Wu et al. [11] studied flame retardation in microencapsulated ammonium polyphosphate and demonstrated a decrease in particle size upon encapsulation and low water absorption. This result is of great interest since the particle size influences the mechanical properties of the coatings. To solve the aforementioned problems, and to investigate lower toxicity coatings, the microencapsulation of dihydrogen phosphate (MAP) as an acid source and low molecular weight tannins as a carbonaceous agent is proposed.

Tannins extracted from Pinus radiata are mixtures of simple phenols such as gallic acid and sugar esters (primarily glucose and gallic/digallic acids). The presence of gallic acid in hydrolyzable tannins makes them ideal raw materials for preparing the coatings [12,13]. Likewise, the presence of hydroxyl groups in tannins, together with their high reactivity compared to resorcinol and phenol, make them ideal candidates 
to produce fire-resistant coatings [14]. Tannins have been used in the production of rigid foams, presenting similar fire-retardant properties to formulations that incorporate specialized additives such as boric acid $\left(\mathrm{H}_{3} \mathrm{BO}_{3}\right)$ or phosphoric acid $\left(\mathrm{H}_{3} \mathrm{PO}_{4}\right)$. Tannins are obtained from waste bark making them a renewable material, and, once extracted, the bark does not lose its calorific capacity and can be used in industrial boilers.

With increasing focus on sustainability in the construction industry, multi-story timber buildings are becoming increasingly popular. the fire issue becomes important since both the wooden claddings and the building construction itself may get involved in the combustion. In this study, the effect of MAP microencapsulation for the generation of a fire-resistant coating, in the presence of tannins (extracted from Pinus radiata as a carbon source), was examined [15]. The mechanical properties of the obtained coatings were compared and their fire resistance was evaluated. The fire-retardant properties of the coatings were evaluated according to ASTM D1360-90a standard methods; this allowed for an indication of the carbonization index and the weight loss of the substrate. The morphologies of the microcapsules were revealed by scanning electron microscopy (SEM) and the changes in the chemical structure were recorded using Fourier Transform Infrared Spectroscopy (FTIR). The mechanical film properties were determined using ISO and ASTM standard methodologies.

\section{Materials and Methods}

The reagents used for the microencapsulation of dihydrogen ammonium phosphate or monoammonium phosphate (MAP) were as follows: 2,4,6-triamine-1,3,5-triazine (melamine), polyvinyl alcohol (PVA), ethanol (96\% v/v), monoammonium phosphate (MAP), formaldehyde $(37 \% \mathrm{v} / \mathrm{v})$, sodium carbonate $\left(\mathrm{Na}_{2} \mathrm{CO}_{3}\right)$, and acetic acid. These were analytical grade and supplied by Merck SA. Pinus radiata tannins were extracted according to the procedure described by Fernández et al. [16] and were used as a carbon source. The bark of Pinus radiata was added to a pilot reactor, and extraction with a mixture of ethanol/water was performed under reduced pressure. The 
extract was subsequently dried in an oven at $40{ }^{\circ} \mathrm{C}$ under reduced pressure until a fine tannin powder was obtained. The appropriate amount of tannin extract or low molecular weight tannins (L-MWT) was dissolved in water to make up the fractions used in this study.

\section{Synthesis of the Prepolymer and Preparation of the MAP Microcapsules}

The prepolymer was synthesized using a modified version of the method reported by $\mathrm{Wu}$ et al. [11]. $2 \mathrm{~g}$ of PVA and $2 \mathrm{~g}$ of melanin were added to a $500 \mathrm{~mL}$ round bottom flask, followed by $100 \mathrm{~mL}$ of distilled water. After the dissolution of the solids, the $\mathrm{pH}$ of the solution was adjusted to 4-5 using acetic acid and was then heated at $90{ }^{\circ} \mathrm{C}$ in an oil bath for 1.5 hours with constant stirring. Subsequently, the $\mathrm{pH}$ of the solution was adjusted to between 8 and 9 using a $10 \%$ (w/v) $\mathrm{Na}_{2} \mathrm{CO}_{3}$ solution, and another $2 \mathrm{~g}$ of melanin was added first, followed by the 5 $\mathrm{mL}$ of formaldehyde $37 \%(\mathrm{v} / \mathrm{v})$. Finally, the obtained mixture was heated at $90{ }^{\circ} \mathrm{C}$ for 1 hour to obtain the prepolymer. MAP microcapsules were prepared as follows: $10 \mathrm{~g}$ of MAP was dispersed in $25 \mathrm{~mL}$ of ethanol by stirring at $1000 \mathrm{rpm}$ for $5 \mathrm{~min}$. Afterward, this solution was added to the prepolymer obtained previously and the $\mathrm{pH}$ of the mixture was adjusted to between 45 using a $10 \%(\mathrm{v} / \mathrm{v}) \mathrm{H}_{2} \mathrm{SO}_{4}$ solution. The resultant solution was heated to $80{ }^{\circ} \mathrm{C}$ with constant stirring at $400 \mathrm{rpm}$ for 3 hours. The solution was then centrifuged at $9000 \mathrm{rpm}$ for $15 \mathrm{~min}$. The obtained microcapsules were filtered and washed with distilled water and dried in an oven at $70{ }^{\circ} \mathrm{C}$ for 24 hours. The obtained solid was denoted as microcapsules of monoammonium phosphate (McMAP).

\section{Coating Formulation, Surface Preparation, and Application Onto Substrates}

Three coatings were produced, each with a different formulation, to determine the effect of the incorporation of microcapsules in active additives on the fire resistance. A water-based acrylic coating was created as a control sample. Microcapsules of MAP (McMAP) were added to this coating at 3 and 9\% w/w ratios. 
The coatings were formulated with $\lambda$ values between 1.37 and 1.51 , where $\lambda$ is the relationship between the pigment volume concentrations (PVC) (50 to 65\%) and the critical pigment volume concentration (CPVC) (36 to 43\%) [17,18]. Table 1 shows the compositions of the coating components. The preparation of the coatings was achieved with a mechanical stirrer and a Cowles-type dispersion propeller operated at speeds of 550-700 rpm. The components were added into a $1 \mathrm{~L}$ plastic container and were constantly agitated by varying the speed of the rotor. Zirconia grinding balls were used to ensure adequate dispersion and to reduce the particle size to a value of 5 Hegman (35 - $40 \mu \mathrm{m}$ ). The surface of the metal substrates (Steel ASTM A36) was prepared according to the established standards of the SSPC (Steel Structures Painting Council. Pittsburgh, USA), applying section SP1 (Solvent Cleaning: removal of all visible oil, grease, soil, drawing and cutting compounds, and other soluble contaminants from steel surfaces with solvent, vapor, cleaning compound, alkali, emulsifying agent, or steam) and SP5 (Metal blast cleaning: when viewed without magnification, the surface shall be free of all visible oil, grease, dust, dirt, mill scale, rust, coating, oxides, corrosion products and other foreign matter).

The wood substrate was dried in an oven at $30{ }^{\circ} \mathrm{C}$ for $48 \mathrm{~h}$ and the adhered powders were superficially cleaned. Due to their high solid content, the formulated coatings were applied onto the substrates using a brush. The thickness of the films was between 150-200 $\mu \mathrm{m}$. The formulated coatings were denoted as: F-Blank (acrylic water-based formulation); F-3\% McMAP (parent formulation + 3\% w/w McMAP); and F-9\% McMAP (blank + $9 \%$ w/w McMAP). In addition, two commercial coatings were tested for comparison. Intumescent FireWall 200 commercial paint; Tricolor (C-INT), and Ignifuga Retardant 77 commercial paint; Chilcorrofin (C-IGN) were used. 
Surfaces, Interfaces and Coatings Technology

Table 1: Specifications of the experimental materials in the blank formulation, F3, and F9. All formulations were prepared in (w/w).

3 and 9 indicate the percentage of McMAP used.

\begin{tabular}{|l|l|l|l|}
\hline Formulations & F-Blank & F-3\% McMAP & F-9\% McMAP \\
\cline { 1 - 3 } Components & & & \\
\hline Water & 27.80 & 27.74 & 27.80 \\
\hline Caolín (Opacit) & 5.12 & 4.75 & 4.75 \\
\hline Calcium carbonate (Hifill) & 13.33 & 12.35 & 3.01 \\
\hline L-MWT & 15.50 & 15.00 & 15.00 \\
\hline McMAP & 0.00 & 3.01 & 9.03 \\
\hline Foamaster MO 2134 & 0.16 & 0.14 & 0.14 \\
\hline Acronal S716 1.09 & 31.00 & 26.00 & 26.00 \\
\hline
\end{tabular}

\section{Characterization and Fire Behavior Tests}

\section{Chemical, Morphological, and Structural Characterization of Microcapsules}

The obtained microcapsules were characterized by Attenuated Total Reflection Fourier Transform Infrared Spectroscopy (ATR-FTIR) using a Spectrum Two kit (Perkin Elmer). Each spectrum was obtained by consecutive scanning at a resolution of $1 \mathrm{~cm}^{-1}$ over the range $4000-500 \mathrm{~cm}^{-1}$. The morphological characteristics of the microcapsules were revealed by Scanning Electron Microscopy (SEM) using a Jeol JSM 6380 LY operated at an acceleration voltage of $20 \mathrm{kV}$. Before SEM analysis, the samples were coated with $50 \mathrm{~nm}$ of gold. Elements present in the microcapsule were qualitatively determined by EnergyDispersive X-ray Spectroscopy (EDS).

\section{Thermogravimetric Analysis (TGA)}

TGA was performed using a Pyris TGA kit (TG 209 F3 Tarsus). Further, $5 \mathrm{mg}$ of the microcapsules was placed in an aluminum crucible and subjected to a heating cycle over the range 25-600 ${ }^{\circ} \mathrm{C}$. The heating rate was $10{ }^{\circ} \mathrm{C} / \mathrm{min}$. The system was purged with nitrogen at a flow rate of $50 \mathrm{~mL} / \mathrm{min}$. 
Surfaces, Interfaces and Coatings Technology

\section{Evaluation of the Properties of the Coatings}

A range of mechanical assessments was conducted on the coated substrates and the commercial coatings according to standard ASTM and ISO testing methodologies. The dynamic viscosity of the coating was measured using a Krebs viscometer in accordance with ASTM D562, while the dry film thickness was measured according to ASTM 6132 using an Elcometer 456 kit. To determine the hydrophobicity of the coating, the contact angle (CA) formed between the coating and a drop of deionized water was determined. The change of the angle over numerous periods of 10 seconds, in static conditions, was evaluated with a Kruss Goniometer model DSA25S controlled by software. The average $\mathrm{CA}$ value was obtained by measuring the same sample in different positions. Mechanical properties were evaluated on metallic substrates with dimensions of $10 \times 10 \times 0.3 \mathrm{~cm}$ for cupping, adhesion, and abrasion tests. Flexibility tests were completed on substrates with dimensions of $15 \times 6 \times 0.3 \mathrm{~cm}$. The standards applied for these tests were as follows: adherence (ASTM D4541 using a PosiTest AT-A kit), abrasion (ASTM D4060 using a Taber Abraser model 5135 kit with a load of $1000 \mathrm{~kg}$ and type CS-10 abrasion wheels), flexibility (ISO 1519 using a BYK cylinder mandrel 5710), and cupping (ISO 1520 using a BYK PF-5405 cupping machine).

\section{Fire Behavior of the Coating}

The fire resistance of the coatings was evaluated using an adapted version of ASTM D1360-90a. A constant flame was applied by a torch, at an inclination of $45^{\circ}$ to the sample, for 180 seconds, taking temperature measurements of the area every 15 seconds. The tests were carried out in triplicates for each formulation. In addition, the weight, width, length, and initial and final thickness of each specimen were recorded throughout the test. Thus, the carbonization index, weight loss, and resistance to temperature increases were evaluated. 


\section{Results and Discussions \\ Chemical, Morphological, and Structural Characterization of Microcapsules}

The FTIR spectra of the encapsulating materials (melamine and PVA) are shown in Figure 1a, while the spectra of the additive to be encapsulated (MAP) and the obtained microcapsules (McMAP) are presented in Figure 1b. Table 2 summarizes the characteristic vibrational bonding signals of the samples. Based on these results, the formation of the McMAPs is evidenced by the disappearance of the stretching bands of the primary amine from the melamine $\left(3500\right.$ and $3440 \mathrm{~cm}^{-1}$ ). This indicates that the reaction with polyvinyl alcohol to form the prepolymer was successful. The appearance of the vinylidene stretching signal at $1664 \mathrm{~cm}^{-1}$ originating from the PVA, as well as the characteristic stretching signal at $3200 \mathrm{~cm}^{-1}$ related to alcohol groups that did not react, confirm the formation of the encapsulating polymer. The encapsulated MAP signals observed at 1256 and $880 \mathrm{~cm}^{-1}$ are ascribed to $\mathrm{P}=\mathrm{O}$ stretching and $\mathrm{P}-\mathrm{O}$ asymmetric stretching vibrations, respectively $[11,19]$.


Figure 1: FTIR spectra of the MAP microencapsulation materials. 
Surfaces, Interfaces and Coatings Technology

Table 2: Infrared vibration signals from reagents and microcapsules.

\begin{tabular}{|l|l|l|l|l|}
\hline \multirow{2}{*}{ Vibration signal } & Melamine & PVA & MAP & McMAP \\
\cline { 2 - 5 } & Wavenumber $\left.\mathbf{( c m}^{-1}\right)$ & \multicolumn{2}{l|}{} \\
\hline $\begin{array}{l}\text { N-H Stretching Primary } \\
\text { Amine }\end{array}$ & $\begin{array}{l}3500 \\
3440\end{array}$ & & & \\
\hline N=C Stretching & 1630 & & & \\
\hline $\begin{array}{l}\text { N-H Bending Primary } \\
\text { Amine }\end{array}$ & 1560 & & & 1560 \\
\hline N-C Stretching & 1020 & & & 1138 \\
\hline O-H Stretching Alcohol & & 3249 & & 3200 \\
\hline $\begin{array}{l}\text { C-H Stretching sp2 sp3 } \\
\text { C=C Stretching alkene }\end{array}$ & & 2936 & & \\
\hline C-H Bending alkane & & 2906 & & 1664 \\
\hline O-H Bending & & 1430 & & \\
\hline C=C Bending alkene & & 1330 & & \\
\hline NH4+ & & 840 & & \\
\hline P=O Stretching & & & 3200 & \\
\hline $\begin{array}{l}\text { P-O Symmetric } \\
\text { Stretching }\end{array}$ & & & 1256 & 1256 \\
\hline $\begin{array}{l}\text { P-O Asymmetric } \\
\text { Stretching }\end{array}$ & & & 1075 & \\
\hline
\end{tabular}

Figure 2 shows the surface morphology, particle diameter, and EDS analysis of the McMAPs. Perfectly spherical microcapsules are observed, with uniform size and no irregularities. The particle size distribution varied between $0.7-1 \mu \mathrm{m}$ with an average diameter of $0.98 \mu \mathrm{m}$ (Figure $2 \mathrm{a}, 2 \mathrm{c}$ ). The diameter of the obtained microcapsules was smaller than that reported by $\mathrm{Wu}$ et al. [11] $(20 \mu \mathrm{m})$. Furthermore, compositional analysis by EDS (Figure 2b) showed characteristic signals for C, N, P, and $\mathrm{O}$; these results confirm the MAP encapsulation. The compositional mapping of the McMAPs is illustrated in Figure 3 ; a homogeneous distribution of each element was seen throughout the microcapsule. The presence of carbon is attributed to the fact that the microcapsule is an organic polymer formed from PVA, melamine, and formaldehyde. The presence of nitrogen results from the incorporation of melamine in the polymer matrix while the observed phosphorus signal is due to 
Surfaces, Interfaces and Coatings Technology

monoammonium phosphate existing both inside the microcapsule and in its wall.

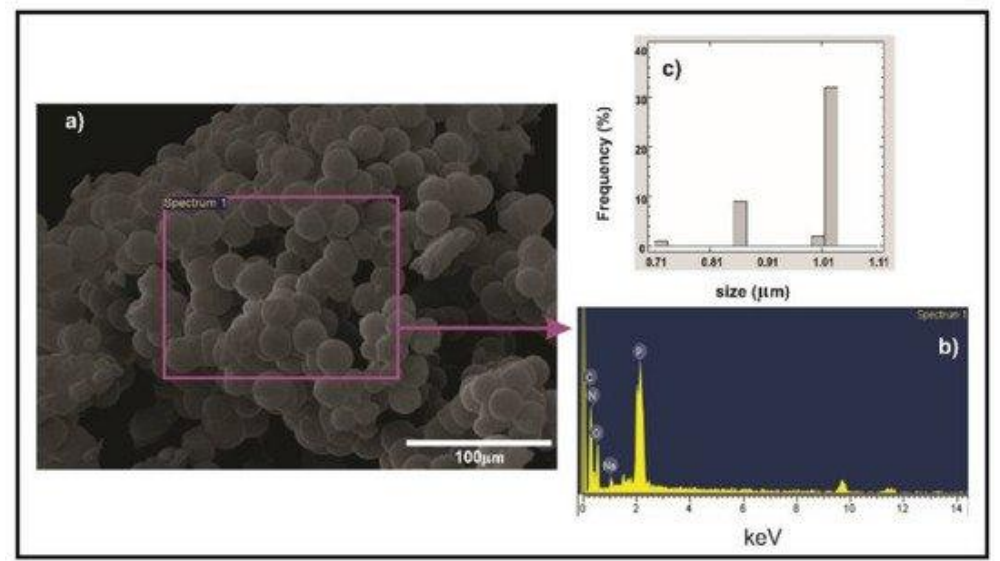

Figure 2: a) SEM micrographs of the McMAP and b) size distribution histogram for the microcapsules. c) EDS analysis.



Figure 3: a) McMAP EDS mapping image and distributions of the various elements that make up the microcapsules: b) carbon $(C), c)$ phosphorus $(P), d)$ nitrogen $(\mathrm{N})$, and e) oxygen $(\mathrm{O})$.

\section{Thermogravimetric Analysis (TGA)}

The thermogravimetric analysis (TGA) curve and Differential Thermogravimetric Analysis (DTG) for the McMAPs are shown in Figure 4 and the decomposition temperatures are reported in Table 3. Only $26.51 \%$ of the initial mass was retained at $600{ }^{\circ} \mathrm{C}$ as revealed in the TGA curve. The observed loss of mass 
corresponds to the thermal degradation of the microcapsules. Upon determining the first derivative DTG of the TGA curve, three clear decomposition processes are identified for McMAP: 40-190 ${ }^{\circ} \mathrm{C}, 200-430{ }^{\circ} \mathrm{C}$, and $430-600{ }^{\circ} \mathrm{C}$. The maximum temperatures of each stage are $103.4{ }^{\circ} \mathrm{C}, 337.2^{\circ} \mathrm{C}$, and $468.2{ }^{\circ} \mathrm{C}$. The first stage of degradation corresponds to the decomposition of PVA and is attributed to the breakdown of ester bonds, the release of non-flammable gases due to the decomposition of melamine, and the evaporation of absorbed water [20,21]. The loss of mass in the second stage is caused by the elimination of $\mathrm{NH}_{3}$ from the melamine units [22]. The final stage of thermal degradation is a result of MAP decomposition in the microcapsules, which generates phosphoric acid and metaphosphoric acid, followed by their dehydration to form phosphorous oxides $\left(\mathrm{P}_{4} \mathrm{O}_{10}\right)$ [21,23].

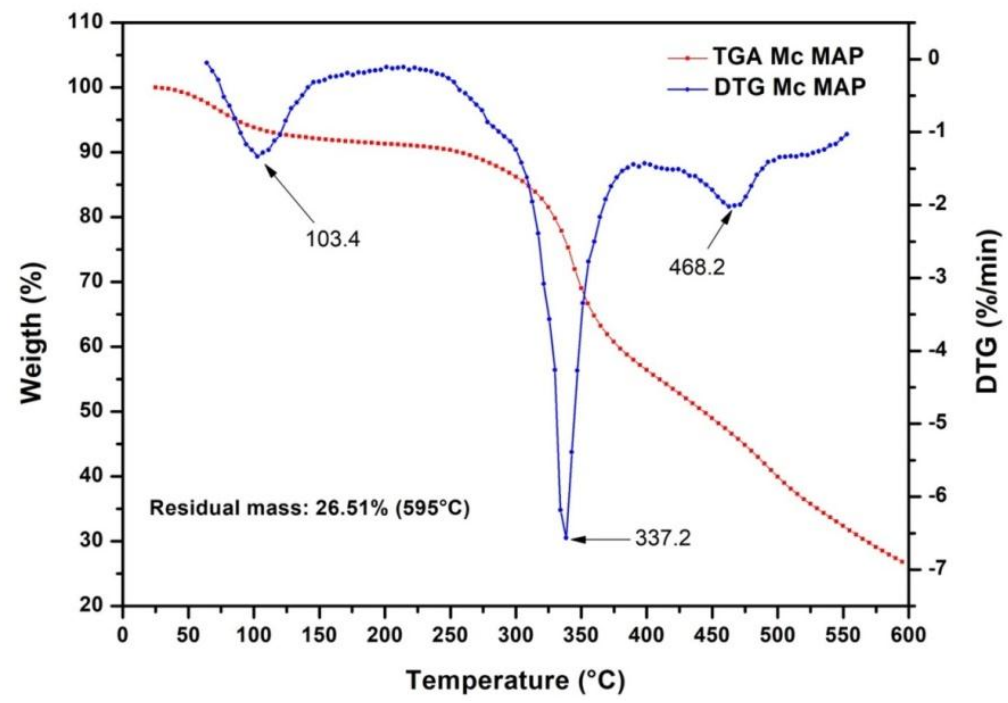

Figure 4: TGA and DTG analysis of the McMAP. 
Surfaces, Interfaces and Coatings Technology

Table 3: Result of decomposition temperature and residual mass of McMAP microcapsules.

\begin{tabular}{|l|l|l|l|l|l|l|l|}
\hline Sample & $\mathbf{T 5 \%}$ & Tmax1 & Tmax2 & Tmax3 & \multicolumn{3}{|c|}{ Residual Mass (\%) } \\
\cline { 5 - 7 } & & & & & $\mathbf{4 0 0}{ }^{\circ} \mathbf{C}$ & $\mathbf{5 0 0}{ }^{\circ} \mathbf{C}$ & $\mathbf{6 0 0}{ }^{\circ} \mathbf{C}$ \\
\hline McMAP & $90.00{ }^{\circ} \mathrm{C}$ & $103.40{ }^{\circ} \mathrm{C}$ & $337.20{ }^{\circ} \mathrm{C}$ & $468.20{ }^{\circ} \mathrm{C}$ & $50 \%$ & $40 \%$ & $27 \%$ \\
\hline
\end{tabular}




\section{Formulation of Coatings with MAP Microcapsules}

The particle sizes for the four formulations described in Table 1 were evaluated using the ASTM D-333 standard. For the reference formulation (F-Blank), a Hegman value of 5 ( $<38.10$ microns) was determined. On the other hand, the formulations that comprised microcapsules (F-3\% McMAP and F-9\% McMAP) presented Hegman values of 4 (<50.80 microns). These results can be explained by the inferior dispersion of the microcapsules in sub-optimal media causing agglomeration. This is the case for intumescent coating formulations that contain high solid content, where satisfactory dispersion of the microcapsules is difficult to achieve. As previously mentioned, the formulations that include microcapsules were produced with a high-volume content of solids, exhibiting PVC contents between $49.90 \%$ and $65.42 \%$, and a $\lambda$ ratio in the range of 1.37 to 1.51 (Table 4). These high PVC contents produce dull coatings and inhibit blistering, however, the permeability and the risk of substrate corrosion increase. For this reason, when industrially applied on steel, these formulations must be coated with a sealant that protects it from corrosive environments. In this work, the resistance of the coatings to weathering was not evaluated, therefore a sealant was not applied.

Table 4: PVC, CPVC and $\lambda$ values for the formulations

\begin{tabular}{|l|l|l|l|}
\hline Formulations & PVC & CPVC & $\boldsymbol{\lambda}$ \\
\hline F-Blank & 49.90 & 36.53 & 1.37 \\
\hline F-3\% McMAP & 58.70 & 39.52 & 1.49 \\
\hline F-9\% McMAP & 65.42 & 43.46 & 1.51 \\
\hline
\end{tabular}

\section{Evaluation of the Mechanical Properties of the Coatings}

Visual inspection of the formulated coatings was conducted using opacity drawdown charts. Figure S1 (a-c) shows the results obtained for the F-Blank and F-3\% McMAP coatings. The formulations show good opacity and covering power when applied on the substrate, with slight changes in the tonality depending on the amount of inorganic filler and tannins present. 
These coatings form a continuous film on the substrate (Figure $\mathrm{S} 1 \mathrm{a}, \mathrm{b}$ ), however, upon further addition of MAP microcapsules (i.e., F-9\% McMAP), agglomerations and cracks were formed on the coating. Results obtained from the dynamic viscosities are shown in Table 5; when the percentage of MAP in the microcapsules increases, the viscosity increases. The F-9\% McMAP sample displayed the highest viscosity with a value of $2407 \mathrm{cp}$. This is because the tannins present in the MAP microcapsules absorb any water in the formulation [11]. This high viscosity is to be expected as the coating has a high solid content, characteristic of intumescent commercial coatings. The high viscosities proved to be problematic for the application of the films onto various substrates, for this reason a brush was used.

Table 5: Evaluation of Film Properties of McMAP Formulations and Commercial Coatings.

\begin{tabular}{|l|c|c|c|c|c|c|c|}
\hline Formulations & $\begin{array}{c}\text { Viscosity } \\
(\mathbf{c p})\end{array}$ & \multicolumn{2}{|c|}{ Steel Substrate } & \multicolumn{2}{c|}{ Wood Substrate } & \multicolumn{2}{c|}{$\begin{array}{c}\text { Contact } \\
\text { Angles }\end{array}$} \\
\cline { 3 - 8 } & & $\begin{array}{c}\text { Dry film } \\
\text { thickness } \\
(\boldsymbol{\mu m})\end{array}$ & $\boldsymbol{\sigma}$ & $\begin{array}{c}\text { Dry film } \\
\text { thickness } \\
(\boldsymbol{\mu m})\end{array}$ & $\boldsymbol{\sigma}$ & $\circ$ & $\boldsymbol{\sigma}$ \\
\hline F-Blank & 381 & 153.5 & 15.40 & 125.1 & 0.07 & 44.56 & 1.10 \\
\hline $\begin{array}{l}\text { F-3\% } \\
\text { McMAP }\end{array}$ & 1743 & 190.8 & 27.00 & 99.1 & 0.49 & 45.38 & 4.78 \\
\hline $\begin{array}{l}\text { F-9\% } \\
\text { McMAP }\end{array}$ & 2402 & 154.9 & 13.40 & 65.55 & 2.68 & 60.27 & 6.02 \\
\hline C-INT & - & 256.86 & 43.35 & 65.73 & 1.73 & 43.42 & 9.06 \\
\hline C-IGN & - & 49.46 & 5.48 & 61.65 & 0.49 & 70.34 & 2.77 \\
\hline
\end{tabular}

Table 5 also shows the dry film thicknesses of the formulations on metal substrates. Figure S1 (d-i) shows the coatings applied on a metal substrate. When two successive coatings were applied on the metallic substrate, the thicknesses of the applied coatings (F-Blank, F-3\% McMAP, and F-9\% McMAP) are between 150 and $190 \mu \mathrm{m}$. Two coats of the commercial paints were also applied on the metallic substrate and these exhibited thicknesses of 256 and $49 \mu \mathrm{m}$ for C-INT and C-IGN, respectively. The difference in thickness is because the commercial intumescent formulation (C-INT) has a high solids content (greater than $65 \%$ ). while the commercial fire-retardant paint (C-IGN) is a varnish with a solid content lower than $30 \%$. Furthermore, the formulated and commercial coatings were applied to wood to 
evaluate fire resistance properties. The thicknesses obtained on wood were lower than those applied on metal. This is most likely due to the absorption of the coating by the open pores of the medium density fiberboard (MDF) wood utilized.

Table 5 shows the contact angle results for the coatings. These results for all evaluated coatings are also shown in Figure 5. The three formulated and the two commercial coatings were hydrophilic in character, presenting contact angle values lower than $90^{\circ}$. The F-9\% McMAP coating displayed was slightly less hydrophilic with an angle of $60.27^{\circ} \pm 6.02$. This performance is attributable to the particle sizes in the McMAPs and the roughness of the coating owing to its high solid content. As the content of microcapsules in the formulations increases, the contact angle also increases. For the F-Blank control coating, the contact angle was $44.56^{\circ} \pm 1.10$, due to the water solubility of the added tannins. Similarly, the commercial coatings present values of $43.42^{\circ}$ and $70.34^{\circ}$ for C-INT and C-IGN, respectively.

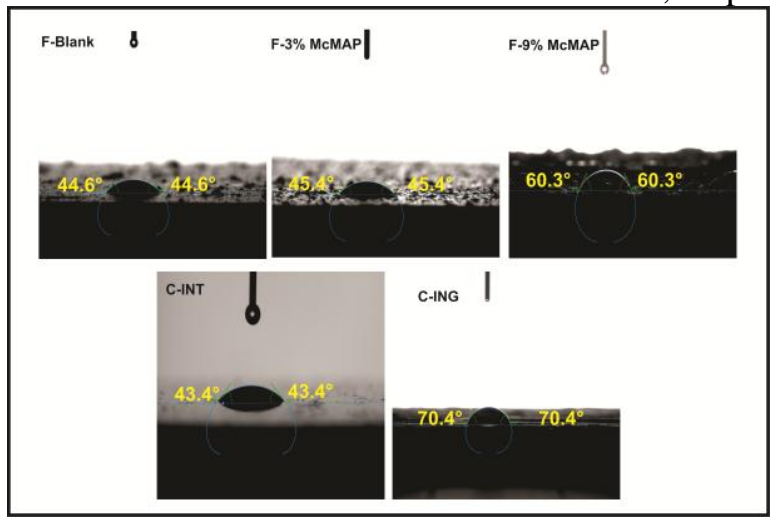

Figure 5: Contact angle of the formulated coatings.

Although the F-9\% McMAP formulation presented the best contact angle as previously mentioned, it did not demonstrate suitable film properties. For this reason, it was not possible to determine its mechanical properties. The mechanical properties of the other coatings (formulated and commercial) were evaluated, and the results are summarized in Table 6. The 3\% McMAP sample showed a slight improvement in the resistance to abrasion with respect to the F-Blank sample and lower losses from mechanical wear were observed with a value of $179.20 \mathrm{mg}$ obtained. 
Surfaces, Interfaces and Coatings Technology

Table 6: Evaluation of the mechanical properties of coatings.

\begin{tabular}{|l|l|l|l|l|l|l|}
\hline \multirow{2}{*}{ Formulations } & Abrasion & Flexibility & Cupping & Adhesion \\
\cline { 2 - 6 } & Wear Index $(\mathbf{m g})$ & Diameter mandrel without fail $(\mathbf{m m})$ & Impact resistence $(\mathbf{m m})$ & Mpa & Type failure \\
\hline F-Blank & 193.40 & 12 & $1.00( \pm 0.68)$ & $1.77( \pm 0.03)$ & $100 \%$ cohesive \\
\hline F-3\% McMAP & 179.10 & 12 & $0.59( \pm 0.04)$ & $0.74( \pm 0.04)$ & $90 \%$ cohesive \\
\hline F-9\% McMAP & - & - & - & - & - & - \\
\hline C-INT & 342.70 & 16 & $1.70( \pm 0.12)$ & $1.06( \pm 0.04)$ & $90 \%$ cohesive \\
\hline C-IGN & 274.22 & 2 & $8.38( \pm 0.04)$ & $3.50( \pm 0.69)$ & $98 \%$ adhesive \\
\hline
\end{tabular}


Figure S2 shows the mass loss of the coatings for the abrasion test. The commercial coatings exhibited the greatest losses, with values of 342.70 and $274.22 \mathrm{mg}$ for the C-INT and C-IGN, respectively. Flexibility tests indicate that formulated coatings performed better than the C-INT, and worse than the C-ING (Figure S3). Because the latter is a varnish, it has excellent flexing properties, unlike high solid content coatings where there is a deficiency of resin in the solid volume (Table 6). The moderate behavior revealed for the F-3\% McMAP is due to the presence of McMAP particles in the formulation. These results agree with those obtained in the abrasion test. The particle size, degree of packing, and continuity of the film are determining factors for obtaining excellent mechanical film properties. A reduction in the adherence by over $50 \%$ was determined; in both formulations the failure mechanism resulted from inadequate cohesion, indicating poor physical interaction within the coating layer. Lower thickness coatings are incomparable with higher thickness coatings in terms of mechanical test

The reference formulation (F-Blank) presented enhanced adhesion to the metallic substrate in comparison to the F-3\% McMAP sample, indicating that the incorporation of the microcapsules produces a relatively negligible negative effect on the adherence to the metal. $1.77 \pm 0.03 \mathrm{MPa}$ of adhesion was determined for F-Blank, compared to $0.74 \pm 0.01 \mathrm{MPa}$ for F-3\% McMAP. Figure S4 shows the results of the adhesion and cupping tests. The cupping test showed similar trends to those observed in adherence tests; the commercial coatings perform better than McMAP formulated coatings. The coatings obtained with F-9\% McMAP failed to obtain film properties and covering power due to the high amount of McMAP, for this reason it was not possible to evaluate their mechanical properties.

\section{Fire Behavior of the Coating}

Fire performance tests for the formulated coatings, were performed on the wood substrate (MDF). Figure 6 shows the specimens after the flame resistance test; a zone of fire advancement is observed on the wood surface. The largest affected area and the highest fire spread rate occurred on 
uncoated wood, followed by F-Blank and F-9\% McMAP. For the F-3\% McMAP formulation, the fire expanded to a lesser degree, behaving similarly to commercial coatings (C-INT and C-IGN). We expected that the fire-retardant effect would be superior with a higher MAP microcapsules content. However, the opposite was seen; this was attributed to the excellent properties of the 3\% McMAP film where its components were homogeneously dispersed, and well-integrated with the resin. Likewise, the presence of L-MWT and McMAP play an important role in generating a sufficient fire-retardant effect. As such, tannins are excellent materials for generating a carbonaceous layer that prevents flame propagation. No significant intumescent behavior (foaming or swelling) was observed in any of the formulated coatings. However, a uniform carbonaceous layer was formed on the wood substrate.



Figure 6: Flame resistance of the formulated coatings.

Figure 7 illustrates the temperature versus time curve of the flame resistance test. This curve represents the increase in temperature of the back of the substrate, where each coating was applied. As can be seen, the flame behavior process occurs in 
three stages. The first stage corresponds to the initial interaction of the fire with the coating; a high-temperature gradient does not occur and it is maintained at $0-1.5^{\circ} \mathrm{C}$ above the initial test temperature, whereas for C-INT, $\Delta \mathrm{T}=0{ }^{\circ} \mathrm{C}$. In the second stage, a dramatic increase in the temperature gradient occurred from 45 to $90 \mathrm{~s}$, until it reached a maximum, due to the partial loss of the coating from the substrate. The maximum obtained temperature gradients were between 10.40 and $32.80{ }^{\circ} \mathrm{C}$. The highest gradient was obtained for F-9\% McMAP, followed by F-3\% McMAP. The final stage is the complete degradation of the coating and the advance of the fire on the wood. A decrease in $\Delta \mathrm{T}$ was observed due to the formation of a superficial carbonaceous layer. This effect was not seen for C-INT, in which only the first two stages were observed. This was due to the formation of foam and/or intumescence of the coating, preventing direct flame propagation and protecting the substrate.

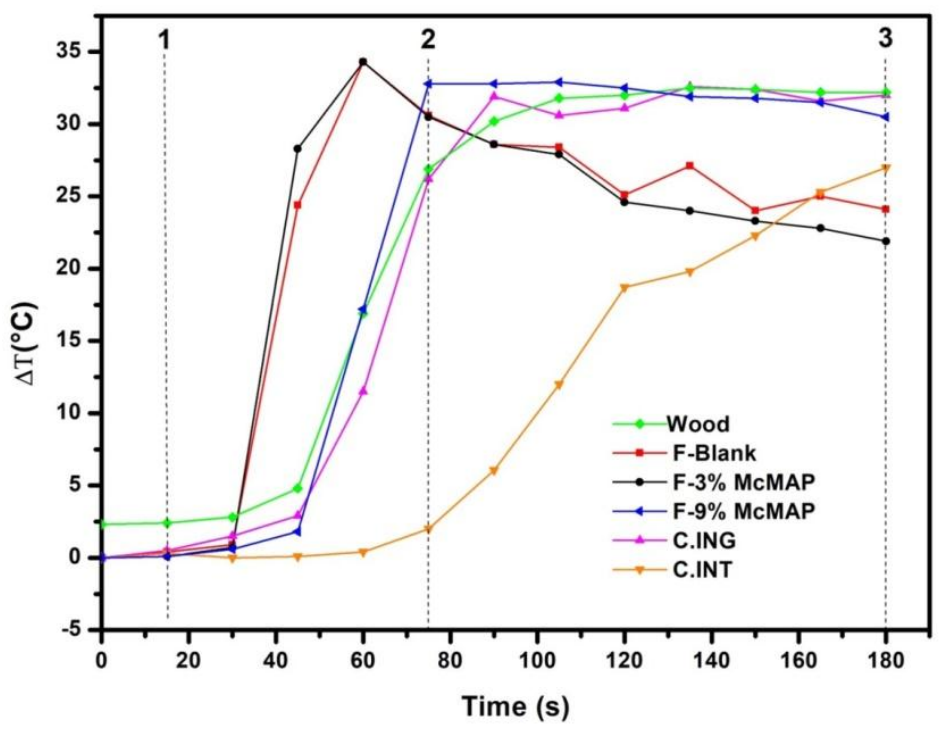

Figure 7: Fire behavior of the formulated coatings.

The results obtained for the carbonization index and the loss of mass in the wood substrate are reported in Table 7 and plotted in Figure 8 . These tests were carried out strictly according to the ASTM D1360-90a (1994) standard methodology. The results 
were limited to the ASTM D1360-90a test setup, it could maybe be different if tested in, e.g., the ISO 5660 Cone Calorimeter at varying heat fluxes or in the full-scale ISO 9705 Room Corner Test, i.e., tests far outside the focus of the present study. Some materials perform well in small scale tests but fail catastrophically in large- or full-scale situations due to high radiant heat flux levels.

The best carbonization index performance was obtained for the F-3\% McMAP coating with a value of $26.86 \%$ determined. This result confirms that superior film and mechanical properties are extremely important to enhance the fire-retardant properties. The carbonization index of uncoated wood was $37.44 \%$ indicating that coatings that contain tannins and McMAP act as flame retardants as they generate a carbonaceous layer that reduces the mass loss of the wood substrate. The C-INT and C-IGN commercial coatings presented carbonization indices of $32.50 \%$ and $39.02 \%$, respectively, demonstrating the superior performance of the F-3\% McMAP. Importantly, the F-3\% McMAP coating displayed the lowest mass loss percentage $(9.05 \%)$, better than the other formulated coatings and the uncoated wood. The mass loss of the F-3\% McMAP coating is greater in comparison to the C-INT, however, its film behavior is superior. Therefore, fire-retardant properties are present in this film. On the other hand, increasing the McMAP content generates greater flame resistance, however, suitable protection is not observed since the carbonization indices and the mass loss percentage are higher. Excellent film properties are essential, as these directly influence the behavior of the coating toward fire. Non-continuous film coatings that contain fissures or cracks allow the heat transport to pass the coating, reducing the fire resistance properties. Although the $3 \%$ formulation was the most efficient, other studies where other percentages of microcapsules are contemplated and tests in Cone calorimetry with different heat fluxes must be carried out, for the complete characterization of the formulas and better understand their resistance against fire. 


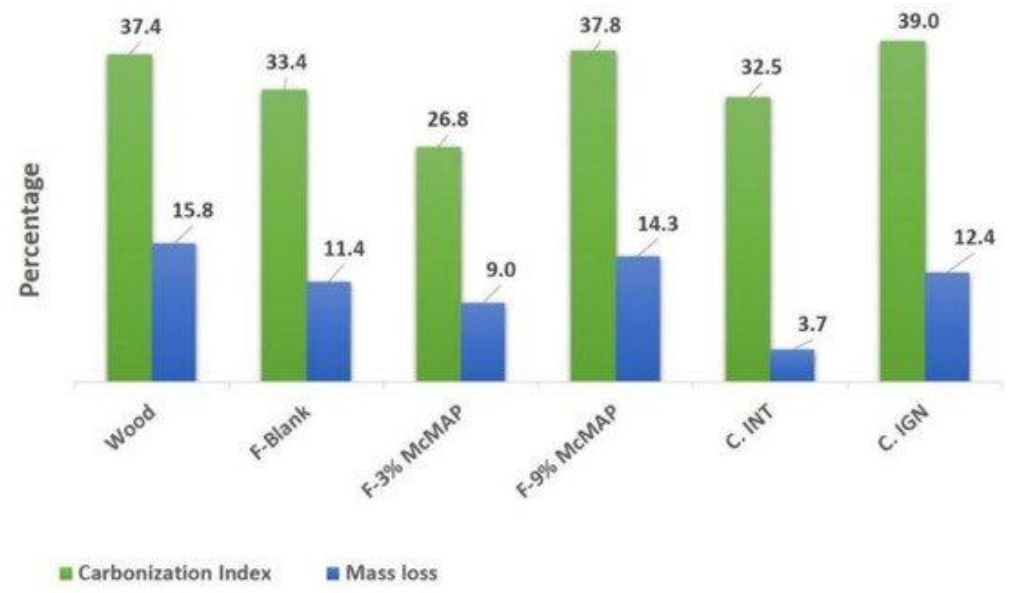

Figure 8: Mass loss and carbonization index of the coatings.

Table 7: Mass Loss and Carbonization Index of Coatings.

\begin{tabular}{|c|c|c|}
\hline Formulations & \% Carbonization Index & \% Mass Loss \\
\hline Uncoated wood & 37.40 & 15.80 \\
\hline F-Blank & 33.40 & 11.40 \\
\hline F-3\% McMAP & 26.80 & 9.00 \\
\hline F-9\% McMAP & 37.80 & 14.30 \\
\hline C-INT & 32.50 & 3.70 \\
\hline C-IGN & 39.00 & 12.40 \\
\hline
\end{tabular}

\section{Conclusions}

We obtained MAP microcapsules through a solution-phase polymeric reaction between melamine and polyvinyl alcohol, as confirmed by FTIR and TGA. Agglomerated microspheres of uniform size were generated, with an average size of $0.98 \mu \mathrm{m}$ and no irregularities. The formulated coatings presented high values of PVC that increased as the incorporation of McMAP increased, affecting the film properties. The three obtained coatings were hydrophilic in character since their contact angles were lower than $90^{\circ}$, however, these angles were higher than those of C-INT and were close to those of C-IGN. The 
mechanical properties of the F-Blank and F-3\% McMAP formulated coating were similar. Increasing the amount of McMAP did not improve the mechanical properties of the coatings; on the contrary, the properties were diminished although not significantly. The coatings containing McMAP exhibited lower temperature gradients indicating greater resistance to fire. The fire progress and substrate temperature were well controlled by the coatings. The F-3\% McMAP coating exhibited superior mechanical and fire resistance properties compared to the F-9\% McMAP coating. Based on this, we conclude that the properties of the coatings deteriorate due to agglomeration upon incorporation of higher amounts of McMAP.

\section{References}

1. Jimenez M, Duquesne S, Bourbigot S. Intumescent fire protective coating: Toward a better understanding of their mechanism of action. Thermochim. Acta. 2006; 449: 16-26.

2. Puri RG, Khanna AS. Effect of cenospheres on the char formation and fire protective performance of water-based intumescent coatings on structural steel. Prog. Org. Coatings. 2016; 92: 8-15.

3. Han Y, Wu H, Zhang W, Zou D, Liu G, et al. Constitutive equation and dynamic recrystallization behavior of as-cast 254SMO super-austenitic stainless steel. Mater. Des. 2015; 69: 230-240.

4. Laoutid F, Bonnaud L, Alexandre M, Lopez-Cuesta JM, Dubois P. New prospects in flame retardant polymer materials: From fundamentals to nanocomposites. Mater. Sci. Eng. R Reports. 2009; 63: 100-125.

5. Wang ZY, Han EH, Ke W. Fire-resistant effect of nanoclay on intumescent nanocomposite coatings. J. Appl. Polym. Sci. 2007; 103: 1681-1689.

6. Liu Z, Dai M, Zhang Y, Gao X, Zhang Q. Preparation and performances of novel waterborne intumescent fire retardant coatings. Prog. Org. Coatings. 2016; 95: 100-106.

7. Shao ZB, Deng C, Tan Y, Chen MJ, Chen L, et al. Flame retardation of polypropylene via a novel intumescent flame 
retardant: Ethylenediamine-modified ammonium polyphosphate. Polym. Degrad. Stab. 2014; 106: 88-96.

8. Cao K, Wu SL, Wang KL, Yao Z. Kinetic study on surface modification of ammonium polyphosphate with melamine. Ind. Eng. Chem. Res. 2011; 50: 8402-8406.

9. Liu Z, Dai M, Hu Q, Liu S, Gao X, et al. Effect of microencapsulated ammonium polyphosphate on the durability and fire resistance of waterborne intumescent fireretardant coatings. J. Coatings Technol. Res. 2019; 16: 135145.

10. Yew MC, Ramli Sulong NH. Fire-resistive performance of intumescent flame-retardant coatings for steel. Mater. Des. 2012; 34: 719-724.

11. Wu K, Song L, Wang Z, Hu Y. Microencapsulation of ammonium polyphosphate with PVA-melamineformaldehyde resin and its flame retardance in polypropylene. Polym. Adv. Technol. 2008; 19: 1914-1921.

12. Montoya LF, Contreras D, Jaramillo AF, Carrasco C, Fernández K, et al. Study of anticorrosive coatings based on high and low molecular weight polyphenols extracted from the Pine radiata bark. Prog. Org. Coatings. 2019; 127.

13. Jaramillo AF, Montoya LF, Prabhakar JM, Sanhueza JP, Fernández K, et al. Formulation of a multifunctional coating based on polyphenols extracted from the Pine radiata bark and functionalized zinc oxide nanoparticles: Evaluation of hydrophobic and anticorrosive properties. Prog. Org. Coatings. 2019; 135: 191-204.

14. Amaral-Labat G, Szczurek A, Fierro V, Stein N, Boulanger $\mathrm{C}$, et al. Pore structure and electrochemical performances of tannin-based carbon cryogels. Biomass and Bioenergy. 2012; 39: 274-282.

15. Celzard A, Fierro V, Amaral-Labat G, Pizzi A, Torero J. Flammability assessment of tannin-based cellular materials. Polym. Degrad. Stab. 2011; 96: 477-482.

16. Bocalandro C, Sanhueza V, Gómez-Caravaca AM, González-Álvarez J, Fernández K, et al. Comparison of the composition of Pinus radiata bark extracts obtained at bench- and pilot-scales. Ind. Crops Prod. 2012; 38: 21-26.

17. Asbeck WK, Loo M. Van Critical Pigment Volume Relationships. Ind. Eng. Chem. 1949; 41: 1470-1475. 
Surfaces, Interfaces and Coatings Technology

18. Rodríguez MT, Gracenea JJ, Saura JJ, Suay JJ. The influence of the critical pigment volume concentration (CPVC) on the properties of an epoxy coating: Part II. Anticorrosion and economic properties. Prog. Org. Coatings. 2004; 50: 68-74.

19. Chupin L, Motillon C, Charrier-El Bouhtoury F, Pizzi A, Charrier B. Characterisation of maritime pine (Pinus pinaster) bark tannins extracted under different conditions by spectroscopic methods, FTIR and HPLC. Ind. Crops Prod. 2013; 49: 897-903.

20. El-Zaher NA, Osiris WG. Thermal and structural properties of poly(vinyl alcohol) doped with hydroxypropyl cellulose. J. Appl. Polym. Sci. 2005; 96: 1914-1923.

21. Giraud S, Bourbigot S, Rochery M, Vroman I, Tighzert L, et al. Microencapsulation of phosphate: Application to flame retarded coated cotton. Polym. Degrad. Stab. 2002; 77: 285297.

22. Kim JH, Kwon DJ, Shin PS, Baek YM, Park HS, et al. The evaluation of the interfacial and flame retardant properties of glass fiber/unsaturated polyester composites with ammonium dihydrogen phosphate. Compos. Part B Eng. 2019; 167: 221-230.

23. Patrick Lim WK, Mariatti M, Chow WS, Mar KT. Effect of intumescent ammonium polyphosphate (APP) and melamine cyanurate (MC) on the properties of epoxy/glass fiber composites. Compos. Part B Eng. 2012; 43: 124-128. 
Surfaces, Interfaces and Coatings Technology

Supplementary Materials:



Figure S1: a-b) Opacity Tests a) F-Blank, b) F-3\% McMAP, c) F-6\% McTannin, d-i) Application of the coating on metal substrate d) F-Blank, e) F$3 \%$ McMAP, f) F-6\% McTannin, g) F-9\% McMAP

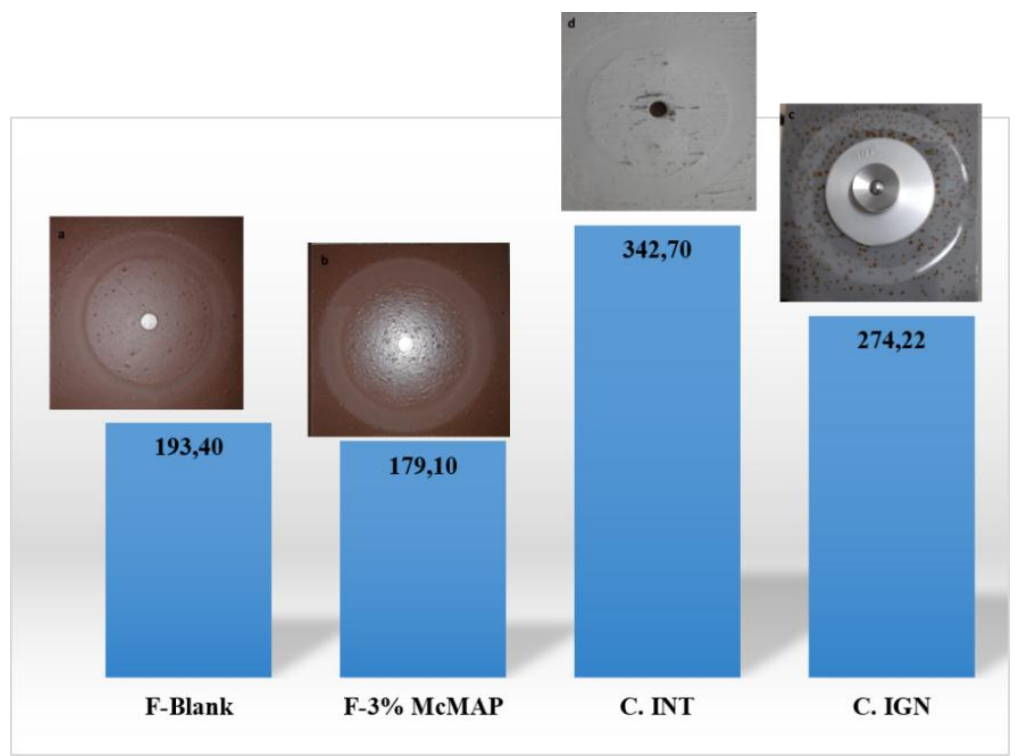

Figure S2: Wear Index a) F-Blank, b) F-3\% McMAP, c) C.IGN and d) C.INT. 
Surfaces, Interfaces and Coatings Technology

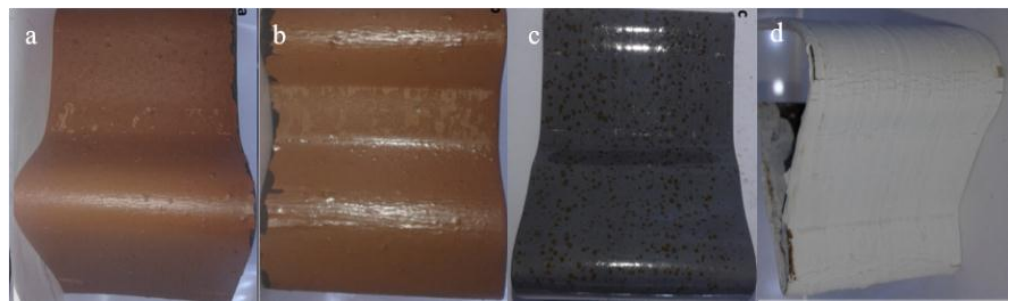

Figure S3: Flexibility test a) F-Blank, b) F-3\% McMAP, c) C.IGN and d) C.INT.

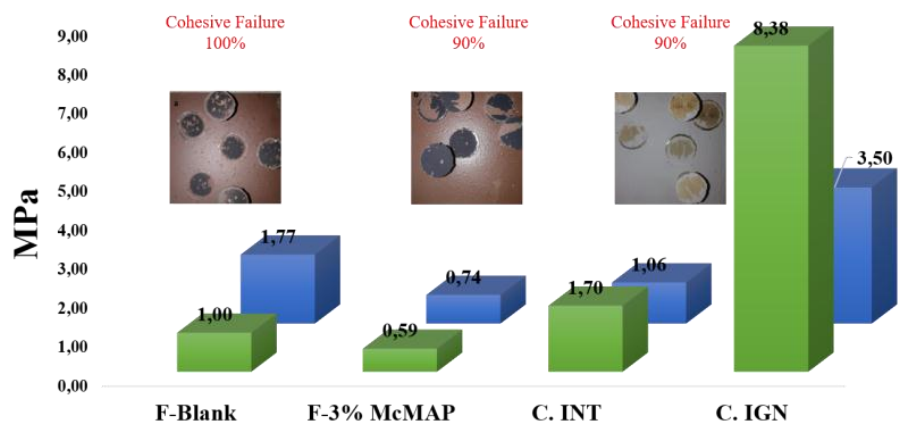

Figure S4: Cupping (green bar) and Pull-off adhesion (blue bar) test on metallic substrate.

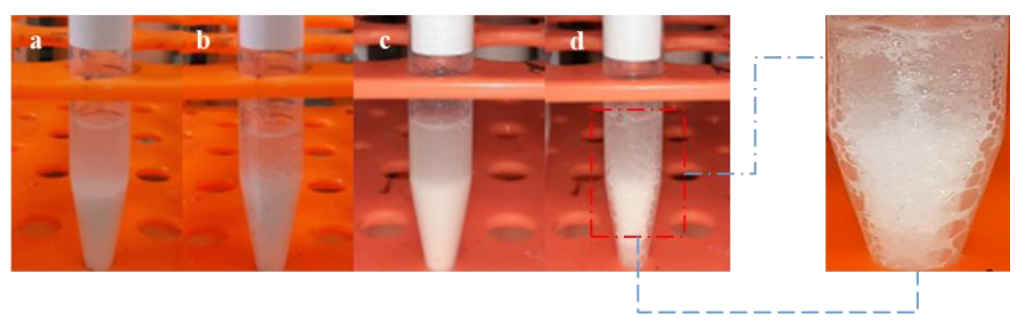

Figure S5. MAP reaction without encapsulation with $\mathrm{Na}_{2} \mathrm{CO}_{3}$ 